\title{
具有二阶细焦点和零特征根 奇点的二次系统
}

张平光蔡䠔林

(浙江大学应用数学系, 杭州)

\section{关锤词 二次系统、细焦点、极限环}

容易证明, 具有二阶细焦点和零特征根奇点的二次系统可以写为

$$
\begin{aligned}
& \frac{d x}{d t}=-y+l x^{2}+m x y+y^{2}, \\
& \frac{d y}{d t}=x(1+a x-y), a>0,
\end{aligned}
$$

其中

$$
m=\frac{a(2 l-1)}{l+1}, l \neq-2,-1,0, \frac{1}{2} .
$$

并且点 $O$ 的第一、第二焦点量分别为 ${ }^{[1]}$

$$
\begin{aligned}
& W_{1} \triangleq m(l+1)-a(2 l-1)-0, \\
& W_{2} \triangleq 2 l m a^{3}(m-5 a) \neq 0 .
\end{aligned}
$$

由文献 [2] p. 755 推论 4 知, (1) 式只能在 $O$ 外围有极限环. $O$ 外围的极限环不可能与 直线 $y=1$ 相交. 于是可作如下一系列变换:

$$
\begin{aligned}
& \xi=\frac{x}{1-y}, \eta=\frac{y}{1-y}, d \tau-(1-y) d t ; \\
& y_{1}=\ln (1+\eta), x_{1}=\xi, d t_{1}=(m-1) d \tau,
\end{aligned}
$$

并且再将 $\left(x_{1}, y_{1}, t_{1}\right)$ 写成 $(x, y, t)$. 于是 (1) 式化为

$$
\frac{d x}{d t}=\Phi(y)-F(x), \frac{d y}{d t}=-g(x),
$$

其中

$$
\begin{aligned}
& g(x)=\frac{x+a x^{2}}{1-m x}, \Phi(y)=e^{y}-1, \\
& F(x)=\frac{(l+1) x^{2}+a x^{5}}{1-m x} .
\end{aligned}
$$

并令

$$
f(x)=F^{\prime}(x)=\frac{x\left[2(l+1)+(3 a-l m-m) x-2 a m x^{2}\right]}{(1-m x)^{2}},
$$




$$
\boldsymbol{G}(x)=\int_{0}^{x} g(\xi) d \xi .
$$

对于一般的系统 (4), 有下述引理 1 和引理 2, 其证明分别见脚注所引的参考文献.

引理 $1^{1)}$ 若曲线 $F\left(x_{2}\right)=F\left(x_{1}\right)$ 与曲线 $G\left(x_{2}\right)=G\left(x_{1}\right)$ 在区域 $D \triangleq\left\{\left(x_{1}, x_{2}\right) \mid x_{02}<\right.$ $\left.x<0,0<x_{1}<x_{01}\right\}$ 内无交点,则方程 (4) 在带域 $x_{02}<x<x_{01}$ 内不存在极限环.

推论 设 $F(x)$ 分别在区间 $x_{02}<x<0$ 和 $0<x<x_{01}$ 内严格单调, 并设曲线 $F\left(x_{2}\right)=F\left(x_{1}\right)$ 与曲线 $f\left(x_{2}\right) / f\left(x_{1}\right)=g\left(x_{2}\right) / g\left(x_{1}\right)$ 在引理 1 的区域 $D$ 内无交点, 则方程 (4) 在带域 $x_{02}<x<x_{01}$ 内不存在极限环.

令 $z=G(x)$, 并以 $x_{i}(z)$ 表示 $z=G(x)\left((-1)^{i+1} x_{i} \geqslant 0, i=1,2\right)$ 的反函数, 则方 程 (4) 可化为

$$
\frac{d z}{d y}=F_{i}(z)-\Phi(y), 0 \leqslant z<z_{0 i}, i=1,2 .
$$

引理 2) 设 $f(x)$ 和 $g(x)$ 在区间 $\left(x_{02}, x_{01}\right)$ 内连续; 当 $x \neq 0$ 时 $x g(x)>0 ; \Phi^{\prime}(y)$ 连续; 当 $y \neq 0$ 时 $y \Phi(y)>0$; 并且满足下述诸条件:

(i) $\Phi^{\prime}(y)>0$;

(ii) 存在 $a_{0}, 0 \leqslant a_{0}<z_{0}=\min \left(z_{01}, z_{02}\right)$, 当 $0<z<a_{0}$ 时 $F_{1}(z) \leqslant F_{2}(z)$, 并且当 $0<z<1$ 时 $F_{1}(z) \neq F_{2}(z)$, 当 $0<z-a_{0} \ll 1$ 时 $\left(F_{1}(z)-F_{1}\left(a_{0}\right)\right)\left(z-a_{0}\right)>0$;

(iii) 如果当 $0<z<z_{02}$ 时 $F_{2}(z)<0$, 则有 $F_{2}^{\prime}(z) \leqslant 0$;

(iv) $F_{1}^{\prime}(z)$ 在 $z>a_{0}$ 上不减(或当 $0<z \ll 1$ 时 $F_{2}(z)>0, F_{2}^{\prime}(z)$ 在 $z>0$ 上不减, 并且 $\left.F_{1}\left(z_{01}-0\right) \leqslant F_{2}\left(z_{02}-0\right)\right)$;

(v) 如果当 $u \geqslant z>a_{0}$ 时 $F_{1}(z)=F_{2}(u)$, 就有 $F_{1}^{\prime}(z) \geqslant F_{2}^{\prime}(u)$.

则方程 (4) 在带域 $x_{02}<x<x_{01}$ 内至多有一个极限环. 若存在, 必为稳定环.

现在证明

定理 具有二阶细焦点和零特征根奇点的二次系统 (1) 有下述 结论: (A) 当 $l \epsilon$ $(-\infty,+\infty) \backslash(-2,-1)$ 时方程 (1) 不存在极限环; (B) 当 $l \in(-2,-1)$ 时方程 (1) 至 多存在一个极限环,若存在, 必包围该二阶细焦点; (C) 当 $0<l+2 \ll 1$ 时方程 (1) 的确 存在极限环.

证 (A) 只要证明当 $l \in(-\infty,-2) \cup(-1,0) \cup\left(0, \frac{1}{2}\right) \cup\left(\frac{1}{2},+\infty\right)$ 时, 满足条件 (3) 并且由 (5) 式确定的系统 (4) 在 $O$ 外围不存在极限环即可. 分 (i) $l>\frac{1}{2}$, (ii) $0<l<$ $\frac{1}{2}$, (iii) $-1<l<0$, (iv) $l<-2$ 四种情形讨论.

(i) $l>\frac{1}{2}$. 此时 $m=\frac{2 l-1}{l+1} a>0$. 将 $f(x)$ 的分子记为 $f_{1}(x)$, 即

$$
\begin{aligned}
f_{1}(x) & =x\left[2(l+1)+(3 a-(l+1) m) x-2 a m x^{2}\right] \\
& =\frac{2 x}{l+1}\left[(l+1)^{2}+a(2-l)(l+1) x-a^{2}(2 l-1) x^{2}\right] .
\end{aligned}
$$

1) 张平光, 方程 $\dot{x}=\Phi(y)-F(x), \dot{y}=-g(x)$ 极限环的唯一性问题 (I).

2) 张平光 $s$ 同上 (II). 
易知 $\left.f_{1}\left(-\frac{1}{a}\right)=-\frac{2 a(2 l-1)}{a(l+1)}<0, f_{1}\left(\frac{1}{m}\right)=\frac{2 l(l+1)^{2}}{(2 l-1)^{2} a}>0,-\frac{1}{a}<0<\frac{1}{m}, f_{2} l-\infty\right)$ $=+\infty, f_{1}(+\infty)=-\infty$. 所以 $f_{1}(x)$ 的三个零点分别小于 $-\frac{1}{a}$, 等于零, 大于 $\frac{1}{m}$. 于是 知 $F(x)$ 在区间 $\left[-\frac{1}{a}, 0\right]$ 上严格单减,在区间 $\left[0, \frac{1}{m}\right]$ 上严格单增. 由引理 1 的推 论 知, 要证本结论成立, 只要证明曲线 $F\left(x_{1}\right)=F\left(x_{2}\right)$ 与曲线 $f\left(x_{1}\right) / g\left(x_{1}\right)=f\left(x_{2}\right) / g\left(x_{2}\right)$ 在区 域 $D^{\prime} \triangleq\left\{\left(x_{1}, x_{2}\right) \mid-1 / a<x_{2}<0,0<x_{1}<1 / m\right\}$ 内不相交即可. 上述两曲线分别为

$$
\begin{aligned}
& \frac{(l+1) x_{1}^{2}+a x_{1}^{3}}{1-m x_{1}}=\frac{(l+1) x_{2}^{2}+a x_{2}^{3}}{1-m x_{1}}, \\
& \frac{2(l+1)+[3 a-(l+1) m] x_{1}-2 a m x_{1}}{\left(1-m x_{1}\right)\left(1+a x_{1}\right)} \\
& \quad=\frac{2(l+1)+[3 a-(l+1) m] x_{2}-2 a m x_{2}}{\left(1-m x_{2}\right)\left(1+a x_{2}\right)} .
\end{aligned}
$$

令 $x_{1}+x_{2}=u, x_{1} x_{2}=v$, 代人 (7) 和 (8) 式, 经计算并约去因子 $\left(x_{1}-x_{2}\right)$, 分别得到

$$
\begin{aligned}
& (l+1) u+a u^{2}-[a+m(l+1)] v-a m v u=0, \\
& 2 l u+[a+(1-l) m] v=0 .
\end{aligned}
$$

区域 $D^{\prime}$ 成为区域 $\widetilde{D} \triangleq\left\{(u, v) \mid-\frac{1}{a m}<v<0,-\frac{1}{a}<u<\frac{1}{m}\right\}$.

设 $l-2$, 则由 (2) 式有 $m=a$, 由 (10) 式得 $u=0$. 再由 (9) 式得 $v-0$. 交点 $(0,0) \bar{E} \widetilde{D}$.

设 $l \neq 2$, 由 (9) 和 (10) 式算得它们的交点为

$$
(u, v)_{1}=(0,0) ;(u, v)_{2}=\left(\frac{(l+2)(l+1)}{(2-l) a}, \frac{(l+2)(l+1)}{(2-l) a^{2}}\right) .
$$

易知 $(u, v)_{1} \in \tilde{D},(u, v)_{2} \bar{E} \widetilde{D}$. 故结论成立.

(ii) $0<l<\frac{1}{2}$, 此时 $m<0$, 易知 $\frac{1}{m}<-\frac{1}{a}<0$. 今证 $F_{1}(x)$ 与 $F_{2}(x)$ 在它们有 定义的公共区间 $0<z<z_{02} \triangleq G\left(-\frac{1}{a}\right)$ 内无交点. 事实上, $F_{2}(0)-F_{1}(0)=0$, 并且,

$$
F_{2}^{\prime}(z)-F_{1}^{\prime}(z)=\frac{a m\left(x_{2}-x_{1}\right)\left[2 l\left(x_{2}+x_{1}\right)+(a+(1-l) m) x_{2} x_{2}\right]}{\left(1-m x_{2}\right)\left(1-m x_{1}\right)\left(1+a x_{2}\right)\left(1+a x_{1}\right)},
$$

这里 $x_{i}-x_{i}(z)(i=1,2)$ 的意义见引理 2 之前的叙述. 由 $G\left(x_{1}\right)-G\left(x_{2}\right)$ 推得

$$
\int_{0}^{x_{2}} \frac{x+a x^{2}}{1-m x} d x=\int_{0}^{x_{1}} \frac{x+a x^{2}}{1-m x} d x \text {. }
$$

于是

$$
\begin{aligned}
\frac{1}{2}\left(x_{2}-x_{1}\right)\left(x_{2}+x_{1}\right) & =\int_{0}^{x_{1}} \frac{(a+m) x^{2}}{1-m x} d x-\int_{0}^{x_{1}} \frac{(a+m) x^{2}}{1-m x} d x \\
& =\int_{x_{2}}^{x_{1}} \frac{(a+m) x^{2}}{1-m x} d x>0 .
\end{aligned}
$$

这里最后一个不等式是由于 $a+m>0,-1 / a \leqslant x_{2}<0<x_{1}<+\infty$, 当 $x \in\left[x_{2}, x_{1}\right]$ 时 $1-m x>0$. 于是由 (12) 式推得 $x_{2}+x_{1}<0$. 又因 $x_{1} x_{2}<0$, 再由 (11) 式推得当 $0<z<$ 
$z_{02}$ 时 $F_{2}^{\prime}(z)<F_{1}^{\prime}(z)$. 故当 $0<z<z_{02}$ 时 $F_{1}(z)$ 与 $F_{2}(z)$ 无公共点. 这就证得方程 (4) 在 $O$ 外围不存在闭轨.

(iii)、(iv) 与 (i) 的证明类似, 从略. (A) 证毕.

(B) 作变换 $(x, y, t) \rightarrow(x,-y,-t)$, 方程 (4) 化为

$$
\frac{d x}{d t}=1-e^{-y}-F(x), \frac{d y}{d t}=-g(x),
$$

其中 $\vec{F}(x)=-F(x)$. 易知 ( $\left.4^{\prime}\right)$ 式满足引理 2 的条件 (i)、（ii）和（iii），其中取 $a_{0}-0$. 余下只需验证 (4') 式满足引理 2 的条件 (iv) 和 (v). 通过计算易知

其中

$$
\left[\frac{f(x)}{g(x)}\right]^{\prime}=\frac{4 a m \omega(x)}{g^{2}(x)}
$$

$$
\omega(x)=l+a l(2-l) x /(l+1) .
$$

易知在区间 $0<x<1 / m$ 内 $\omega(x)<0$, 从而 $f(x) / g(x)$ 在区间 $0<x<1 / m$ 内不增. 条 件 (iv) 满足. 再验证 (v). 由脚注 2) 的引文的附注知, 只需验证对任何 $c>0$, 曲线 $F\left(x_{2}\right)=$ $F\left(x_{1}\right)$ 与 $G\left(x_{2}\right)=G\left(x_{1}\right)+c$ 在区域 $\left\{\left(x_{1}, x_{2}\right) \mid 0 \leqslant x_{1}<1 / m,-1 / a<x_{2}<0\right\}$ 内至多有 一个交点. 此与 (A) 的 (i) 的证明类似,从略. 条件 (v) 满足. 情形 (B) 证毕.

(C) 由第一、第二焦点量公式 (3) 及第三焦点量 ${ }^{[1]}$

$$
W_{3} \triangleq-2 l m a^{4}\left(2 a^{2}+l+2\right)
$$

知, 当 $m=(2 l-1) a /(l+1)$ 及 $l=-2$ 时, $W_{1}=W_{2}=0, W_{3}=40 a^{7}>0$. 当 $m=$ $(2 l-1) a /(l+1)$ 及 $0<l+2 \ll 1$ 时, $W_{1}=0, W_{2}<0$. 于是推知 $O$ 外围存在极限环. (C) 证毕. 定理证毕.

推论 设二次系统有一个一阶焦点量为零的奇点和一个有零特征根的奇点, 则该二次 系统至多有一个极限环。若存在此极限环必包围细焦点.

证明略.

文献 [3] 是研究具有二重零特征根的二次系统, 显然该文与本文互不相包.

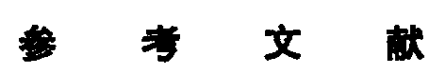

[1] 叶产谦,极限环论,上海科学技术出版社, 1984 。

[2] 陈兰苏、王明淑,数学学报, 22(1979),6:751-758.

[3] Coppel, W. A., Bull. Austral. Math. Soc., 38(1988),1-10. 